\title{
Liver dual arterial blood supply maintains liver regeneration: Analysis of signaling pathways in rats
}

\author{
JIAN-LIANG QIAO ${ }^{1}$, JUAN SUN ${ }^{2}$, JUN LI $^{1}, \mathrm{JUN}^{-J I N G ~ Z H A N G}{ }^{1}$ and XING-KAI MENG ${ }^{1}$ \\ ${ }^{1}$ Department of Hepatobiliary Surgery, The Affiliated Hospital of Inner Mongolia Medical University, \\ Hohhot 010050; ${ }^{2}$ Department of Immunology, Inner Mongolia Medical University, Hohhot 010110, P.R. China
}

Received April 11, 2017; Accepted October 18, 2017

DOI: $10.3892 / \mathrm{mmr} .2017 .7961$

\begin{abstract}
Liver dual arterial blood supply (LDABS) could increase blood supply to the liver and maintain normal liver regeneration in patients with compromised portal vein. The current study attempted to examine the underlying molecular mechanisms. Male Sprague-Dawley rats randomly received partial hepatectomy $(\mathrm{PH})$ alone or $\mathrm{PH}$ followed by LDABS. Liver regeneration was assessed by histological examination, liver function and liver regeneration rate (LRR). Whole-genome oligo microarray analysis was used to compare gene expression profile between rats receiving $\mathrm{PH}$ and rats receiving $\mathrm{PH}$ plus LDABS. Key genes identification was validated using a MAPK signaling polymerase chain reaction (PCR) array. The extent of liver regeneration in rats receiving $\mathrm{PH}$ plus LDABS was comparable to that in rats receiving $\mathrm{PH}$ alone. The differentially expressed genes were enriched in 12 signaling pathways in two groups. MAPK signaling pathway, NF-kappa B signaling pathway, and Toll-like receptor signaling pathway were involved in LDABS-mediated liver regeneration, with Retinoblastoma $1(R b l)$, Cyclin D1, Cyclin-dependent kinase 4, Mitogen-activated protein kinase 10 (Mapk10) and CAMP responsive element binding protein 1 genes in the initiation phase, Kirsten rat sarcoma viral oncogene homolog (Kras), tumor protein 53, MYC proto-oncogene, BHLH transcription factor, Cyclin E1 and Heat shock protein family B (small) member 1 genes in the proliferation phase, Kras, Rbl, Jun proto-oncogene, AP-1 transcription factor subunit, Cyclin D2 and Mapk10 genes in the termination phase were identified as key genes in LDABS-mediated liver regeneration using MAPK signaling PCR array analysis.
\end{abstract}

Correspondence to: Professor Jun-Jing Zhang or Xing-Kai Meng, Department of Hepatobiliary Surgery, The Affiliated Hospital of Inner Mongolia Medical University, No. 1 Tongdao North Street, Hohhot 010050, P.R. China

E-mail: zjj7811@gmail.com

E-mail: mxk6788@gmail.com

Key words: liver dual arterial blood supply, partial hepatectomy, liver regeneration, signaling pathway

\section{Introduction}

The liver has dual blood supplies: the portal vein and the hepatic artery. When the portal vein is compromised, by thrombosis for example, capacity of the liver regenerate is limited. Liver dual arterial blood supply (LDABS) is a surgical procedure that shunts arterial blood to the portal vein system for enhancing liver blood supply to maintain liver regeneration. LDABS is distinct from arterioportal fistula (1) as well as simple portal vein arterialization, in which the portal vein is the only route to supply blood to the liver because of injury, thrombosis or surgical removal of the proper hepatic artery $(2,3)$.

LDABS could extend the usefulness of portal vein arterialization (4) and has been used to manage portal vein thrombosis before and after orthotopic liver transplantation (OLT) or auxiliary liver transplantation (ALT) $(5,6)$. LDABS could maintain hepatic function and morphology in some patients for as long as 3 years (7).

The mechanisms by which LDABS maintains liver regeneration are not fully understood, but clearly differ substantially from liver regeneration that occurs after $\mathrm{PH}$. Unlike $\mathrm{PH}$, LDABS involves loss of blood supply from the portal vein, changes in blood components and hemodynamics of the portal vein system, and changes in liver anatomy.

In the current study, we used whole-genome oligo microarray analysis to examine the molecular changes in a rat model of PH plus LDABS. Key genes identification was validated using a MAPK signaling PCR array. The results of this study may help expand our understanding of signaling pathways that underlie liver regeneration and provide a firmer foundation for further developing LDABS as a clinical tool.

\section{Materials and methods}

The study protocol was approved by the Laboratory Animal Ethics Committee of Inner Mongolia Medical University. Briefly, male Sprague-Dawley rats (Vital River Laboratory Animal Technology; Beijing, China) randomly received $\mathrm{PH}$ alone or PH followed by LDABS ( $=20 /$ group). Rats were sacrificed immediately at 0, 6, 48 and $168 \mathrm{~h}$ after the surgery ( $n=5$ per time point). Remnant liver was collected for whole genome oligo microarrays. Trunk blood $(2 \mathrm{ml})$ was collected from the infrahepatic inferior vena cava. 
$P H$. The operation was performed by two surgeons using clean but not sterile technique under a microscope (Leica M525 F20, Germany). Rats were fasted for $24 \mathrm{~h}$ prior to operation. Anesthesia was induced by inhalation of 3-4\% isoflurane, and maintained by inhalation of $1-2 \%$ isoflurane. After anesthesia induction, a median incision was cut on the abdomen. The left and middle lobes of the liver were resected using bloodless hepatectomy (Fig. 1), as previously reported (8).

$L D A B S$. After the left and middle lobes of the liver were resected, the infrahepatic caval vein was isolated. The right renal artery was isolated and clamped. The right renal vein was isolated and ligated with a 1-0 silk suture adjacent to the infrahepatic caval vein. The right kidney was removed. The pyloric vein was ligated with an 8-0 nylon suture and divided; the proximal and distal ends of the portal vein were clamped, and the main portal vein was isolated and dissected transversely at the midpoint. The right renal artery was anastomosed with the proximal end of the portal vein using a MicroRenathane catheter (length: $10 \mathrm{~mm}$; inner diameter: $0.5 \mathrm{~mm}$ ). Then the clamps were removed successively from the portal vein and right renal artery to allow portal vein arterialization.

The distal end of the portal vein was anastomosed with the right renal vein, as described (9). The clamps were removed successively from the right renal vein and portal vein to allow portacaval shunt (Fig. 1). After irrigation with warm lactated Ringer's solution $(5 \mathrm{ml})$, the abdomen was closed.

Rat body temperature was maintained at $36^{\circ} \mathrm{C}$ using a heating blanket throughout the procedure. Rats received daily low molecular weight heparin (50 IU/ml, s.c.). Antibiotics and analgesics were not used.

Evaluation of liver regeneration. Liver regeneration was assessed by visual inspection with naked eyes as well as microscopic examination of hepatic cells and portal areas following hematoxylin-eosin staining. Alanine transaminase (ALT) and albumin (ALB) levels in serum were measured using an automated analyzer. Regeneration is quantitatively expressed as liver regeneration rate (LRR), based on liver weight (LW), and calculated as follows:

$$
L R R=\frac{L W \text { at autopsy }- \text { estimated residual LW at time of surgery }}{\text { Resected LW }} \times 100 \%
$$

Residual liver weight at the time of surgery was estimated based on the assumption that the left and middle hepatic lobes account for 70 percent of total liver weight (8).

Whole-genome oligo microarray analysis. Total RNA was harvested from the liver using TRIzol (Invitrogen) and the RNeasy kit (Qiagen); this procedure included a DNase digestion step. RNA was quantified using a Nanodrop ND-1000 apparatus (NanoDrop Technologies, Wilmington, DE, USA). RNA quality was verified with denaturing gel electrophoresis. Samples were amplified and labeled using the Agilent Quick Amp labeling kit and hybridized using an Agilent 4x44K whole-genome oligo microarray in Agilent SureHyb Hybridization Chambers. After hybridization and washing, microarray slides were scanned using the Agilent DNA microarray scanner (G2505B). Text files of results were extracted using Agilent Feature Extraction Software (version 10.5.1.1) and imported into Agilent GeneSpring GX software (version 10.0). Genes differentially expressed between the 2 groups were defined as $>2.0$ fold-change and $\mathrm{P}<0.05$ between the 2 groups at each time point. Identified genes were analyzed using the KEGG PATHWAY Database (http://www.genome. jp/kegg/). Two-sided Fisher's exact test was used to classify enriched pathways. Enrichment was defined by $(a / n) /(A / N)$, where $a$ represents the number of target genes; $n$, the total number of genes in the particular pathway; $A$, the total number of differentially expressed genes in all the pathways; and $N$, the total number of genes in all the pathways.

MAPK signaling PCR array analysis. Regeneration-related genes differentially expressed between the 2 groups were analyzed using a rat MAPK signaling PCR Array (SuperArray Bioscience, Frederick, Maryland, USA). RNA was extracted and converted to first-strand cDNA using the RT ${ }^{2}$ First Strand Kit. The template was added to an instrument-specific, ready-to-use $\mathrm{RT}^{2} \mathrm{SYBR}$ Green qPCR Master Mix. The resulting mixture was added to 96-well PCR array plate $(25 \mu \mathrm{l} / \mathrm{well})$ pre-loaded with gene-specific primer sets $(25 \mu 1)$. PCR was performed and threshold cycle $(\mathrm{Ct})$ values for all genes on each PCR array were calculated using instrument-specific software. Fold-changes in gene expression between the 2 groups were calculated using the DDCt method. Gene interaction networks were constructed using Pathway Studio (Ariadne Genomics, NX Amsterdam, the Netherlands).

Statistical analysis. Continuous variables are expressed as mean \pm standard deviation (SD). Statistical analyses were carried out using SPSS 18.0 (IBM, Chicago, IL, USA). Homogeneity of variance was assessed using the $\mathrm{F}$ test and the collecting dates were analyzed by repeated measurement analysis of variance. $\mathrm{P}<0.05$ was defined as the threshold of significance.

\section{Results}

Histological examination. Both groups showed initial hepatic congestion and subsidence over time; the remnant liver in the LDABS group was brighter than that the control group (Fig. 1). Liver weight increased to a similar extent in the 2 groups. Neither group showed evidence of macroscopic necrotic findings, such as petechia and ecchymoses.

Liver tissue from the control group showed integral hepatic lobules, hepatic cords radially arranged around a central vein, distinct hepatic sinuses, and absence of inflammatory cell infiltration in and around portal areas (Fig. 2). In contrast, liver tissue from the LDABS group showed markedly dilated hepatic sinuses, peaking at $6 \mathrm{~h}$ postoperatively. Red blood cells (RBCs) were present to various extents in selected sinuses, but without RBC deposits or thrombosis. Interlobular veins were dilated. Normal bile canaliculi were visible in portal areas. Pyknosis and necrosis were observed in a small fraction of hepatocytes in the central zone. Hepatic cords appeared normal. Tissue edema and vacuolar degeneration of hepatocytes increased over time, peaking at $48 \mathrm{~h}$ postoperatively. Hepatic sinus dilation and congestion decreased gradually over time.

Liver function. ALT level increased to a peak at $6 \mathrm{~h}$ after the surgery, and then gradually returned to the baseline in both 
A

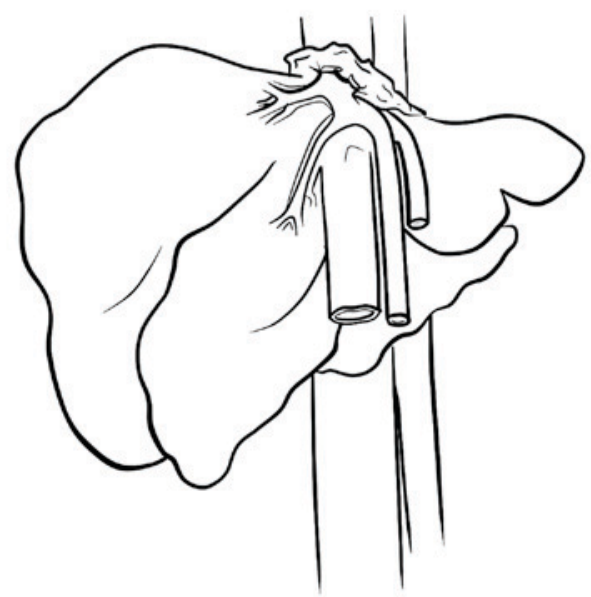

$\mathrm{C}$

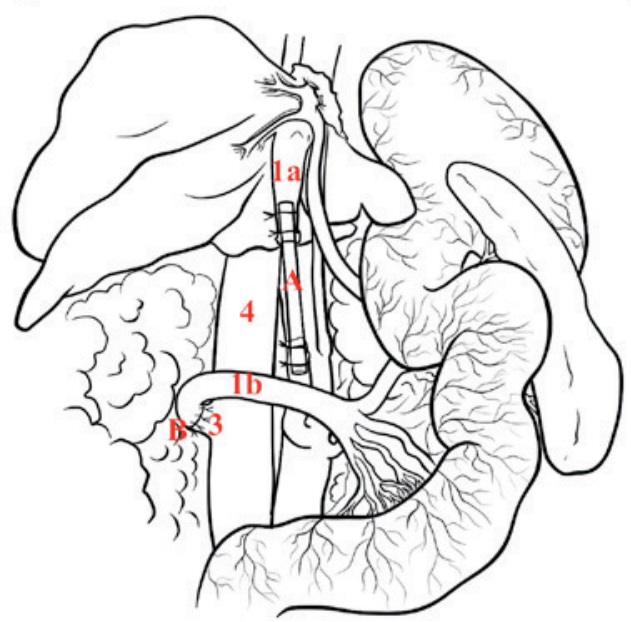

B

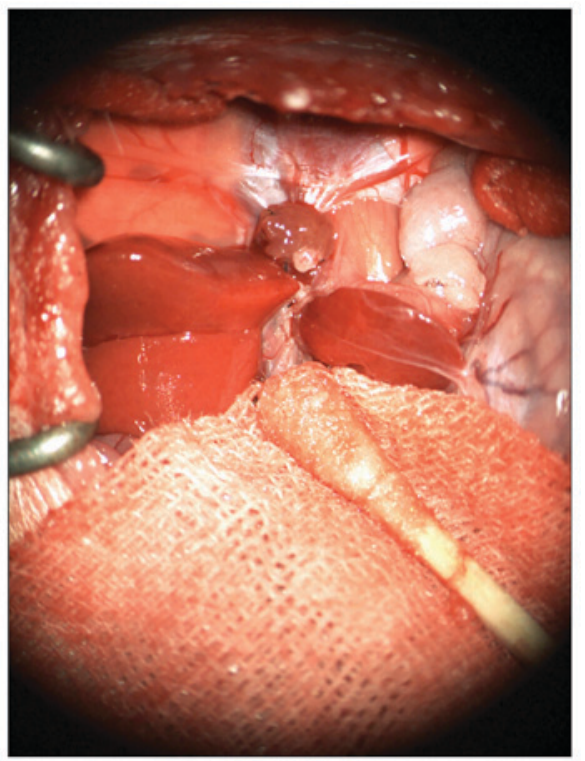

$\mathrm{D}$

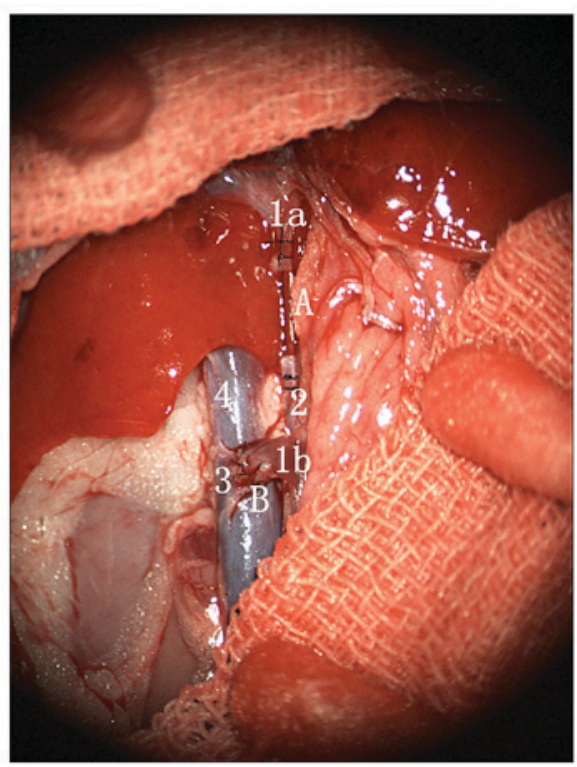

Figure 1. (A) A schematic illustration of PH using using bloodless hepatectomy. (B) Representative photo of PH during surgery. (C) A schematic illustration of LDABS using the artery and vein of the right kidney in rats. (D) Representative photo of LDABS during surgery. A, portal vein arterialization using a stent technique to connect the proximal end of the portal vein and the right renal artery; $\mathrm{B}$, a portocaval shunt created by end-to-end anastomosis of the distal end of the portal vein and the right renal vein; 1a, proximal end of portal vein; $1 \mathrm{~b}$, distal end of portal vein; 2 , right renal artery; 3 , right renal vein; 4, infrahepatic caval vein; PH, partial hepatectomy; LDABS, Liver dual arterial blood supply.

groups (Fig. 3). ALT was lower in the LDABS group at $6 \mathrm{~h}$ $(782.9 \pm 59.9$ vs. $411.2 \pm 54.6, \mathrm{P}<0.05)$, but not other time points $(\mathrm{P}>0.05)$. ALB level decreased to a trough at $6 \mathrm{~h}$, and then gradually returned to the baseline in both groups (Fig. 4). ALB level was comparable between the 2 groups at all time points $(\mathrm{P}>0.05)$.

LRR. LRR gradually increased over time, reaching $100 \%$ by $168 \mathrm{~h}$ in both groups (Fig. 5), and LRR did not differ between the 2 groups at all time points $(\mathrm{P}>0.05)$.

Differential gene expression. In order to reveal molecular mechanisms of LDABS, gene expression was compared between rats treated by $\mathrm{PH}$ in the absence or presence of LDABS using whole-genome oligo microarray analysis of hepatic tissue. After limiting the results to genes showing $>2$-fold difference between the two conditions (with $\mathrm{P}<0.05$ ), pathway analysis was used to identify signaling pathways altered in LDABS-mediated regeneration (Table I). Wayne chart analysis identified up-regulated genes in arginine and proline metabolism signaling pathway at all three time points, and down-regulated genes in the following 11 signaling pathways (Fig. 6): cytokine-cytokine receptor interaction, malaria, HTLV-I infection, NF-kappa B signaling pathway, Chagas disease (American trypanosomiasis), amoebiasis, rheumatoid arthritis, Toll-like receptor signaling pathway, MAPK signaling pathway, toxoplasmosis, and ECM-receptor interaction. In all these signaling pathways only the MAPK signaling pathway, NF-kappa B signaling pathway, and Toll-like receptor signaling pathway which regulated post $\mathrm{PH}$ 
Table I. Whole-genome oligo microarray analysis of genes differentially expressed between rats with PH alone vs. with PH plus LDABS.

\begin{tabular}{lcccc}
\hline $\begin{array}{l}\text { Time } \\
\text { point, } h\end{array}$ & $\begin{array}{c}\text { Number of } \\
\text { upregulated genes }\end{array}$ & $\begin{array}{c}\text { Signaling pathways } \\
\text { enriched with } \\
\text { upregulated genes }\end{array}$ & $\begin{array}{c}\text { Number of } \\
\text { downregulated genes }\end{array}$ & $\begin{array}{c}\text { Signaling pathways } \\
\text { enriched with } \\
\text { downregulated genes }\end{array}$ \\
\hline 6 & 2,267 & 39 & 1,566 & 38 \\
48 & 1,653 & 37 & 928 & 36 \\
168 & 1,235 & 18 & 668 & 42 \\
\hline
\end{tabular}

PH, partial hepatectamy; LDABS, liver dual arterial blood supply.
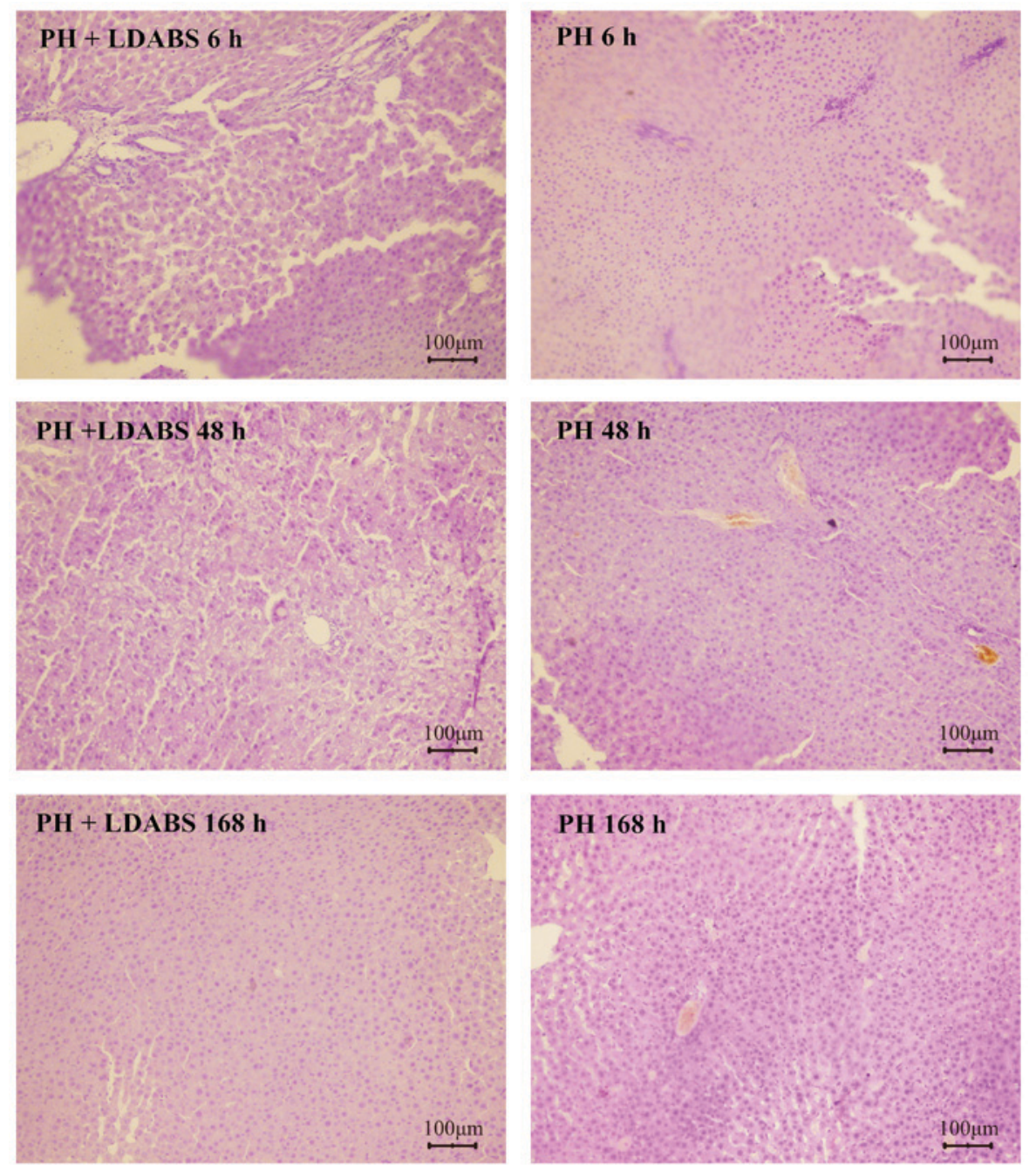

Figure 2. Microscopic analysis of liver tissue from rats with PH alone vs. PH plus LDABS. Sections were prepared at different points and stained with hematoxylin-eosin. Magnification, x200. PH, partial hepatectomy; LDABS, Liver dual arterial blood supply.

liver regeneration were involved in LDABS-mediated liver regeneration.

MAPK signaling pathway involvement using PCR array analysis. Since our study identified MAPK signaling pathway were involved in LDABS-mediated liver regeneration, we validated our results for 84 genes associated with MAPK signaling pathway using MAPK signaling pathway PCR array analysis. The results confirmed that several changes were expressed at different levels ( $>2$ fold-change) in the absence or presence of LDABS (Table II), The genes list is as follows:

A. Time point $6 \mathrm{~h}$

Number of upregulated genes (16): Ccnd1, Cdk4, Cdkn1c, Col1a1, Creb1, Egr1, Map4k1, Mapk10, Mapk11, Mapk12, Mapk8ip1, Mef2c, Mknk1, Mos, Nfatc4 and Rb1. Number of downregulated genes (5): Cdkn1a, Dlk1, Map2k3, Mapk14 and Mapk7. 
Table II. MAPK signaling pathway polymerase chain reaction array analysis to confirm differential expression of mitogen-activated protein kinase signaling-related genes in liver dual arterial blood supply-mediated liver regeneration.

\begin{tabular}{lcc}
\hline $\begin{array}{l}\text { Time } \\
\text { point, h }\end{array}$ & $\begin{array}{c}\text { Number of } \\
\text { upregulated genes }\end{array}$ & $\begin{array}{c}\text { Number of } \\
\text { downregulated genes }\end{array}$ \\
\hline 6 & 16 & 5 \\
48 & 10 & 5 \\
168 & 3 & 14 \\
\hline
\end{tabular}

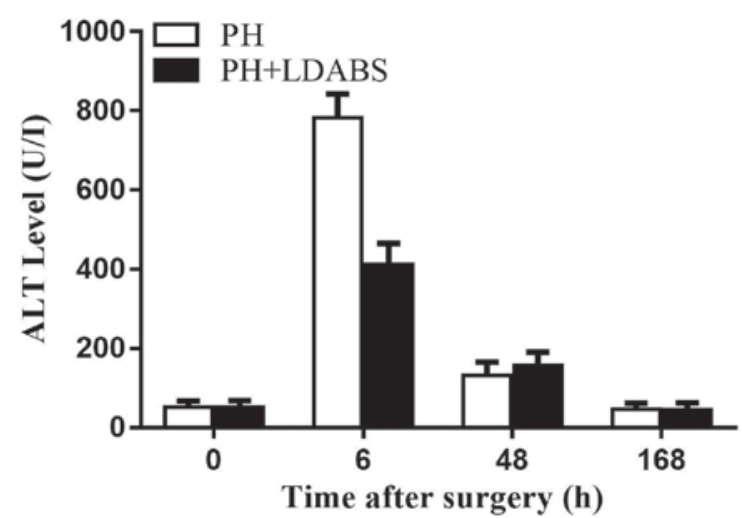

Figure 3. ALT levels (U/I) in rats with PH vs. with PH plus LDABS. Levels were determined at different points. PH, partial hepatectomy; LDABS, Liver dual arterial blood supply; ALT, Alanine transaminase supply.

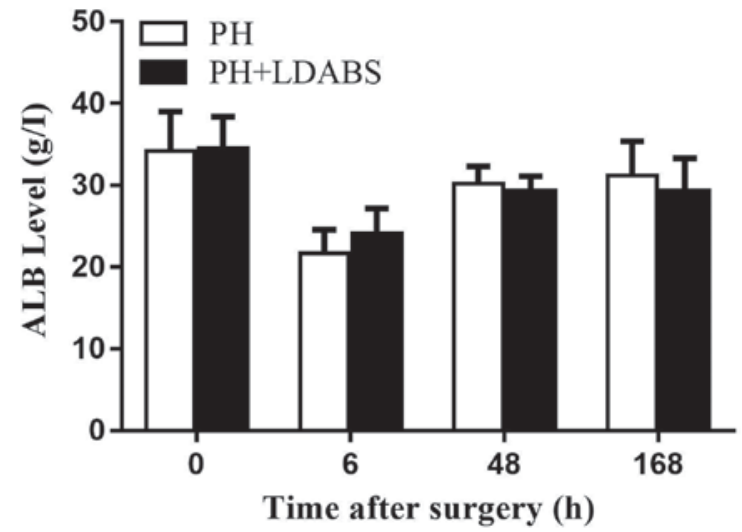

Figure 4. ALB levels (g/I) in rats with PH vs. with PH plus LDABS. Levels were determined at different points. PH, partial hepatectomy; LDABS, Liver dual arterial blood supply; ALB, albumin.

\section{B. Time point $48 \mathrm{~h}$}

Number of upregulated genes (10): Dlk1, Egr1, Ets2, Fos, Hspb1, Jun, Kras, Myc, Actb and Ldha. Number of downregulated genes (17): Ccne1, Cdkn1a, Col1a1, Egr1, Ets1, Fos, Hspb1, Kras, Ksr1, Mapk10, Mapk13, Mapk8ip3, Mef2c, Mos, Myc, Nfatc4 and Tp53.

C. Time point $168 \mathrm{~h}$

Number of upregulated genes (3): Map2k6, Nfatc4, Rb1. Number of downregulated genes (14): Ccnd2, Cdk6, Cdkn2a,

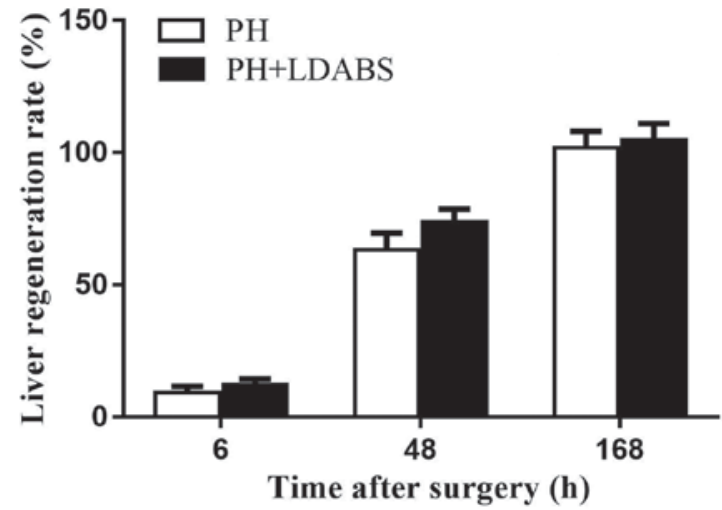

Figure 5. Liver regeneration rate (\%) in rats with $\mathrm{PH}$ vs. with $\mathrm{PH}$ plus LDABS. The rate was determined at different points. $\mathrm{PH}$, partial hepatectomy; LDABS, Liver dual arterial blood supply.

Cdkn2d, Dlk1, E2f1, Egr1, Fos, Jun, Kras, Ksr1, Map4k1, Mapk10 and Mapk8ip3.

The up regulation of genes was significantly increased at $6 \mathrm{~h}$, while the down regulation of genes was significantly increased at 48 and $168 \mathrm{~h}$. These findings suggest that MAPK signaling pathway is involved in post $\mathrm{PH}$ regeneration in the absence or presence of LDABS, but that the pathway functions differently (or to a different extent) in the presence of LDABS.

Network analysis to identify key genes. Our microarray results and data from the literature were used to construct an interaction network of genes differentially expressed in LDABS-mediated liver regeneration (Figs. 7-9), Combined the list of genes in Table II, Rbl, Ccnd1, Cdk4, Mapk10 and Crebl genes in the initiation phase, Kras, Tp53, Myc, Ccnel and Hspbl genes in the proliferation phase, Kras, Rb1, Jun, Ccnd2 and Mapklo genes in the termination phase were identified as key genes in LDABS-mediated liver regeneration using MAPK signaling PCR array analysis.

\section{Discussion}

Portal vein thrombosis (PVT) represents a major barrier for liver transplantation because diffuse PVT is widely considered a contraindication for liver transplantation. In 1995, Erhard et al (5) described what would become known as the LDABS, procedure that allowed a patient with advanced liver cirrhosis to undergo OLT. All components of the portal vein system in this patient showed diffuse PVT, with the exception of a segment of inferior mesenteric vein, and embolectomy proved unsuccessful. Blood flow to the transplanted liver was restored by connecting the portal vein and hepatic artery of the liver to the abdominal aorta of the recipient via the donor iliac blood vessels. Subsequent portal hypertension was addressed by portacaval shunt. Normal liver function with no fibrotic changes (confirmed by hepatic biopsy) was maintained for 12-month follow up. Nearly a decade later, a modified LDABS procedure involving was carried out patient with advanced liver cirrhosis scheduled to undergo OLT (10). This patient showed diffuse PVT in the portal vein system. Blood flow to the transplanted liver was restored by connecting the 

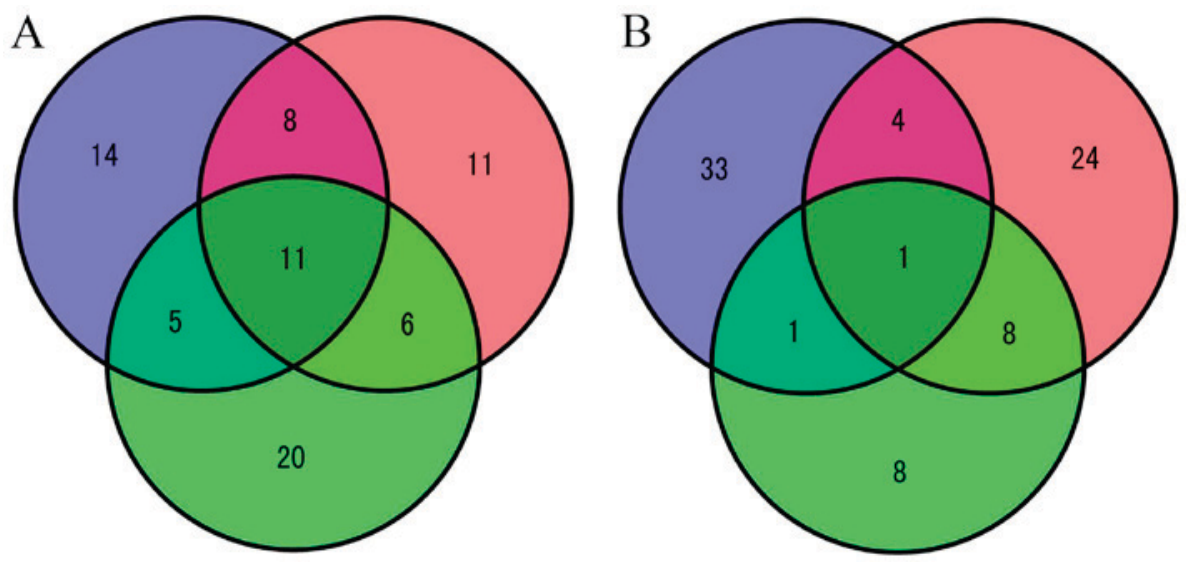

Figure 6. Wayne chart analysis showing (A) up-regulated or (B) down-regulated signaling pathways in liver dual arterial blood supply-mediated liver regeneration.

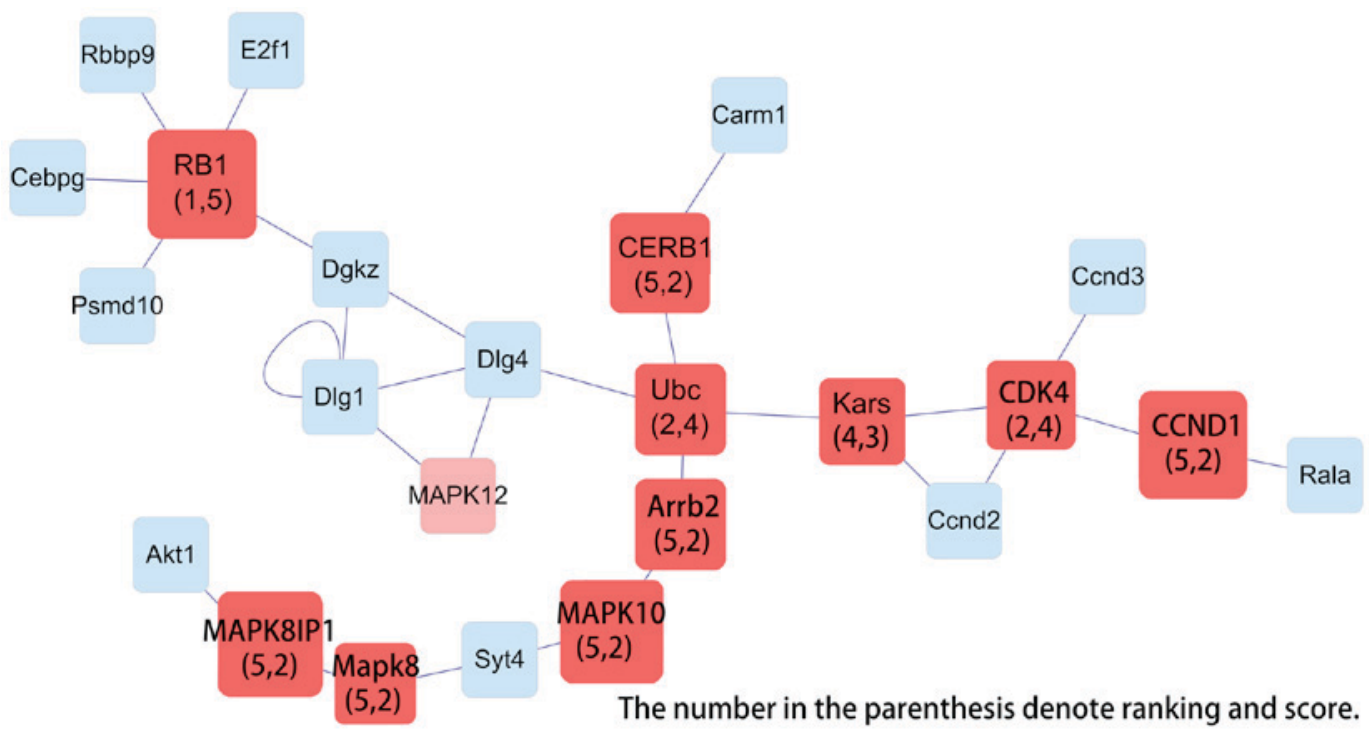

Figure 7. Interaction network of genes differentially expressed in liver dual arterial blood supply-mediated liver regeneration and identification of key genes involved at 6 h. Rb1, Retinoblastoma 1; Ubc, ubiquitin C; CDK4, Cyclin-dependent kinase 4; Kras, Kirsten rat sarcoma viral oncogene homolog; MAPK, Mitogen-activated protein kinase; Arrb2, Arrestin Beta 2; CCND1, Cyclin D1; CREB1, CAMP responsive element binding protein 1 genes in the initiation phase.

portal vein to the hepatic artery and the hepatic artery of the donor liver to the abdominal aorta. That patient survived for $>20$ months.

In a literature research, we identified 10 patients with PVT who underwent LDABS since 1995 to reconstruct blood flow in a transplanted liver (5,7,10-13). All patients survived for long periods except two deaths caused by liver necrosis or liver fibrosis, possibly due to portal vein hyperperfusion. These results suggest that LDABS can enable OLT upon diffuse PVT.

Evidence suggests that LDABS can also be effective for managing PVT after OLT, helping to prevent or defer repeat transplantation. In a case report, a patient received two OLTs, the first transplantation due to biliary atresia and the second due to transplanted liver lymphoma (6). On day 3 after the second transplantation, diffuse PVT was detected in the portal vein system. After LDABS that connected the donor portal vein with the recipient abdominal aorta via donor iliac blood vessels, liver function recovered over despite of eventual death due to multiple organ failure.
Several cases have also been reported in which LDABS enabled ALT to be performed in patients who were otherwise ineligible because of acute hepatic failure, congenital metabolic liver disease or benign end stage liver disease. Of the 7 cases of LDABS-based ALT that we found in the literature (5,6,14-16), two showed completely regenerated host liver and survived the removal of the transplanted liver; one underwent OLT because of liver failure 10 days after ALT; and four died of multiple organ failure, cytomegaloviral pneumonia or sepsis, none of which was associated with the surgical technique. These results suggest that LDABS-based ALT can substantially benefit carefully selected patients because the transplanted liver can substitute for the failed host liver and effectively provide hepatic function.

The fact that previous studies of LDABS in patients are limited to individual case studies justifies the use of preclinical animal models to explore the procedure in more detail. A previous study from this laboratory showed similar extent of liver regeneration in rats with $\mathrm{PH}$ plus LDABS vs. $\mathrm{PH}$ 


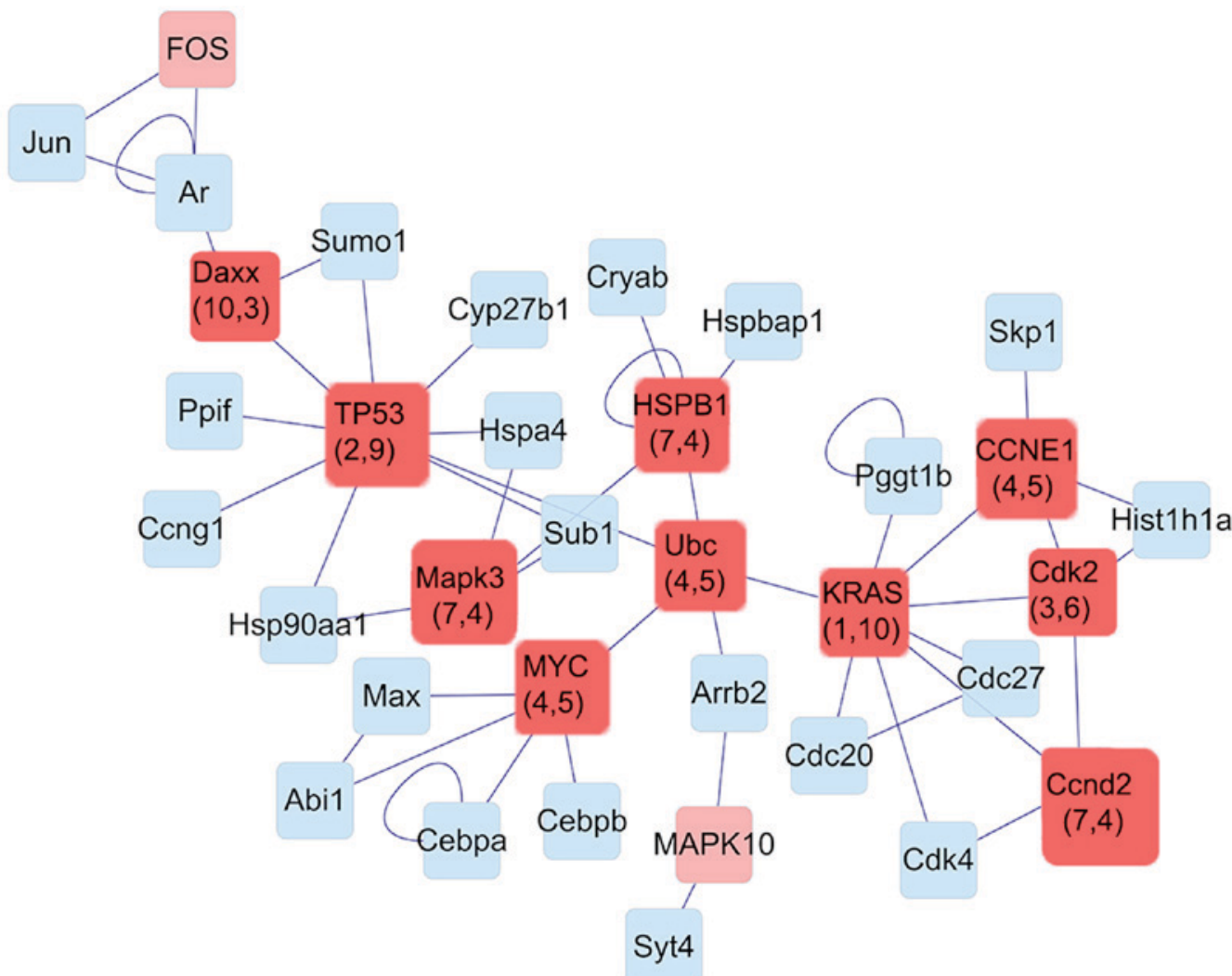

The number in the parenthesis denote ranking and score.

Figure 8. Interaction network of genes differentially expressed in liver dual arterial blood supply-mediated liver regeneration and identification of key genes involved at 48 h. Kras, Kirsten rat sarcoma viral oncogene homolog; TP53, tumor protein 53; Cdk2, cyclin-dependent kinase 2; Ubc, ubiquitin C; MYC, MYC proto-oncogene, BHLH transcription factor; CCNE1, Cyclin E1; Mapk3, Mitogen-activated protein kinase 3; Ccnd2, Cyclin D2; HSP1, Heat shock protein family B (small) member 1 genes in the proliferation phase; Daxx, Death domain associated protein.

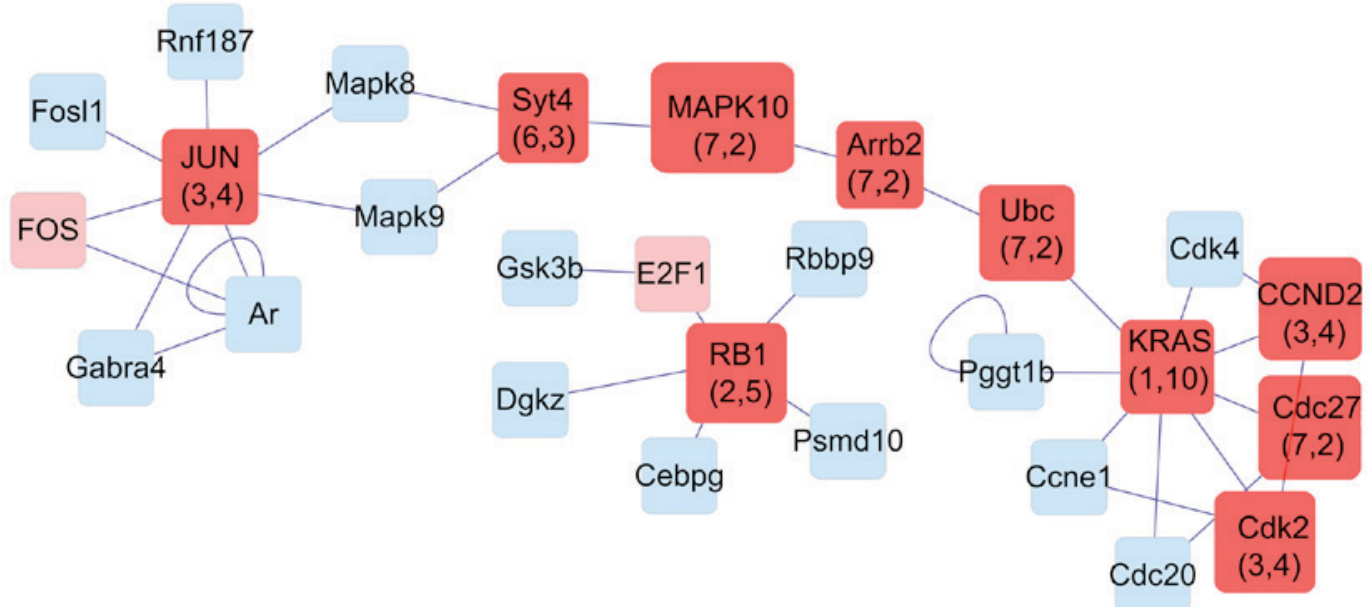

The number in the parenthesis denote ranking and score.

Figure 9. Interaction network of genes differentially expressed in liver dual arterial blood supply-mediated liver regeneration and identification of key genes involved at $168 \mathrm{~h}$. Kras, Kirsten rat sarcoma viral oncogene homolog; Rb1, Retinoblastoma 1; JUN, Jun proto-oncogene, AP-1 transcription factor subunit; Cdk2, Cyclin-dependent kinase 2; CCND2, Cyclin D2; Syt4, synaptotagmin; MAPK10, Mitogen-activated protein kinase 10; Arrb2, Arrestin Beta 2; Cdc27, cell-division cycle 27.

alone (17). The present study further verified these previous findings.

The molecular mechanisms of LDABS-mediated liver regeneration are unclear. Lack of understanding in this aspect poses an obstacle to further development and clinical implementation of the technique. Liver regeneration requires strict spatiotemporal coordination of numerous hepatic genes activated by cytokines and growth factors, which are in turn 
regulated by various signaling pathways (18-20), including pathways dependent on MAPK, JAK/STAT, NF-kappa B, Notch, Hedgehog, Toll-like receptor, CXCR, Wnt, and RHO. The up- or down-regulation of genes in these signaling pathways may promote or suppress hepatocellular proliferation or apoptosis. Previous work from our group suggested that the mechanisms behind LDABS-mediated liver regeneration differ from regeneration after PH alone. Specifically, fluorescent quantitative RT-PCR in our previous study revealed differential expression of TNF- $\alpha$, HGF and TGF- $\beta 1$ in the liver of rats with PH alone vs. with PH plus LDABS (21).

The rat model of $70 \% \mathrm{PH}$ was used by many investigators to study liver regeneration. After $70 \%$ liver resection in 7-10 days, the residual liver tissue was completely recovered. Liver regeneration consists of three stages: the initiation phase (2-6 $\mathrm{h}$ after $\mathrm{PH})$, the proliferation phase (12-72 $\mathrm{h}$ after $\mathrm{PH}$ ) and the termination phase (120-168 $\mathrm{h}$ after $\mathrm{PH})$. So we chose three time points $(6,48,168 \mathrm{~h})$ to represent the different stages of liver regeneration.

The present study combined whole-genome oligo microarrays with MAPK signaling PCR arrays. The results implicated differentially expressed genes were enriched in 12 signaling pathways using pathway analysis, but only the MAPK signaling pathway, NF-kappa B signaling pathway, and Toll-like receptor signaling pathway which regulated post $\mathrm{PH}$ liver regeneration were involved in LDABS-mediated liver regeneration, we speculate that these three pathways contribute to liver regeneration on different time points or act on different downstream targets.

MAPK (mitogen activated protein kinase) belongs to a class of intracellular serine/threonine protein kinase. Downstream genes was activated by the activation of the MAPK signaling pathway through continuous enzymatic reaction (MAPK kinase (MAP4Ks), MAPK kinase (MAP3Ks), MAPK kinase (MAP2Ks), MAPKs (ERKs, JNK and P38)) to promote cell proliferation, differentiation and apoptosis (22). Results showed the up regulation of genes was significantly increased at 6 and $48 \mathrm{~h}$, while the down regulation of genes was significantly increased at $168 \mathrm{~h}$ among 84 genes of MAPK signaling pathway gene chip. It is illustrated that there is dual regulation of MAPK signaling pathway on liver regeneration at different stages.

Network analysis identified the Rbl, Ccndl, $C d k 4$, Mapkl0 and Crebl genes in the initiation phase, Kras, Tp53, Myc, Ccnel and Hspbl genes in the proliferation phase, Kras, Rbl, Jun, Ccnd2 and Mapk10 genes in the termination phase were identified as key genes in LDABS-mediated liver regeneration using MAPK signaling PCR array analysis. However, the experiments of this study were based only to gene expression, not the protein expression, the roles of these genes should be further investigated, for example, using western blotting and knockout mice.

In conclusion, MAPK signaling pathway play an important role in regulation of LDABS-mediated liver regeneration. $R b 1$, Ccndl, Cdk4, MapklO and Crebl genes in the initiation phase, Kras, Tp53, Myc, Ccnel and Hspbl genes in the proliferation phase, Kras, Rb1, Jun, Ccnd 2 and Mapk10 genes in the termination phase were identified as key genes in LDABS-mediated liver regeneration using MAPK signaling PCR array analysis, the up- or down-regulation of these genes may promote or suppress liver regeneration in the LDABS.

\section{Acknowledgements}

This study was supported by the National Natural Science Foundation of China (81260073,81560113); Natural Science Foundation of Inner Mongolia Autonomous Region, China (2014MS0850); Major Program of the Affiliated Hospital of Inner Mongolia Medical University (NYFYZD2014006); Scientific Research Program in Colleges and Universities of Inner Mongolia Autonomous Region, China (NJZY113). Program for Young Talents of Science and Technology in Universities of Inner Mongolia Autonomous Region (NJYT-17-A15).

The whole-genome oligo microarray and MAPK signaling PCR array were carried out by Shanghai Kangchen Bio-tec, ltd.

\section{References}

1. Iwaki T, Miyatani H, Yoshida Y, Matsuura K and Suminaga Y: Gastric variceal bleeding caused by an intrahepatic arterioportal fistula that formed after liver biopsy: A case report and review of the literature. Clin J Gastroenterol 5: 101-107, 2012.

2. Qiu J, Wu H, Prasoon P and Zeng Y: Portal vein arterialization in hilar cholangiocarcinoma: One case report and literature review. Eur J Gastroenterol Hepatol 24: 229-232, 2012.

3. Melandro F, Lai Q, Levi Sandri GB, Guglielmo N, Di Laudo M, Morabito V, Pretagostini R, Berloco PB and Rossi M: A case of portal vein arterialization after a liver transplant. Exp Clin Transplant 11: 287-289, 2013.

4. Tsivian M, Neri F, Prezzi D, Puviani L, Pacile V, Bertelli R, Cavallari G, Mattioli B, Bianchi E, Piras GL, et al: Portal vein arterialization in hepatobiliary surgery and liver transplantation. Transplant Proc 39: 1877-1878, 2007.

5. Erhard J, Lange R, Giebler R, Rauen U, de Groot H and Eigler FW: Arterialization of the portal vein in orthotopic and auxiliary liver transplantation. A report of three cases. Transplantation 60: 877-879, 1995.

6. Charco R, Margarit C, López-Talavera JC, Hidalgo E, Castells L, Allende H, Segarra A, Moreíras M and Bilbao I: Outcome and hepatic hemodynamics in liver transplant patients with portal vein arterialization. Am J Transplant 1: 146-151, 2001.

7. Settmacher U, Stange B, Schaser KD, Puhl G, Glanemann M, Steinmüller T, Heise M and Neuhaus P: Primary permanent arterialization of the portal vein in liver transplantation. Transpl Int 16: 430-433, 2003.

8. Martins PN, Theruvath TP and Neuhaus P: Rodent models of partial hepatectomies. Liver Int 28: 3-11, 2008.

9. Qiao JL, Wang ZY, Zhang JJ and Meng XK: Assisting suspension triangulated continuous suture technique for microvascular anastomosis in rat portocaval shunt. Microsurgery 35: 166-167, 2015.

10. Nivatvongs S, Sirijindakul B and Nontasoot B: Portal vein arterialization for liver transplantation with extensive portomesenteric vein thrombosis: A case report. Transplant Proc 36: 2267-2268, 2004.

11. Aspinall RJ, Seery JP, Taylor-Robinson SD and Habib N: Comments on 'arterialization of the portal vein in orthotopic and auxiliary liver transplantation'. Transplantation 62: 1375-1376, 1996.

12. Stange B, Glanemann M, Nussler NC, Bechstein WO, Neuhaus P and Settmacher U: Indication, technique, and outcome of portal vein arterialization in orthotopic liver transplantation. Transplant Proc 33: 1414-1415, 2001.

13. Ott R, Böhner C, Müller S, Aigner T, Bussenius-Kammerer M, Yedibela S, Kissler H, Hohenberger W, Reck T and Müller V: Outcome of patients with pre-existing portal vein thrombosis undergoing arterialization of the portal vein during liver transplantation. Transpl Int 16: 15-20, 2003.

14. Erhard J, Lange R, Rauen U, Scherer R, Friedrich J, Pietsch M, de Groot $\mathrm{H}$ and Eigler FW: Auxiliary liver transplantation with arterialization of the portal vein for acute hepatic failure. Transpl Int 11: 266-271, 1998.

15. Margarit C, Bilbao I, Charco R, Lázaro JL, Hidalgo E, Allende E and Murio E: Auxiliary heterotopic liver transplantation with portal vein arterialization for fulminant hepatic failure. Liver Transpl 6: 805-809, 2000. 
16. Lange R, Rauen U, Janssen H, Erhard J and de Groot $\mathrm{H}$ : Temporary heterotopic auxiliary liver transplantation with arterialization of the portal vein as treatment of acute liver failure. Transpl Int 20: 473-474, 2007.

17. Zhang JJ, Niu JX, Dong CX and Meng XK: Portal vein arterialization used in partial hepatectomy maintains liver regeneration. Sci Res Essays 6: 6325-6330, 2011.

18. Riehle KJ, Dan YY, Campbell JS and Fausto N: New concepts in liver regeneration. J Gastroenterol Hepatol 26 (Suppl 1): S203-S212, 2011.

19. Nowatari T, Fukunaga $\mathrm{K}$ and Ohkohchi N: Regulation of signa transduction and role of platelets in liver regeneration. Int $\mathrm{J}$ Hepatol 2012: 542479, 2012.
20. Taub R: Liver regeneration: From myth to mechanism. Nat Rev Mol Cell Biol 5: 836-847, 2004.

21. Niu JX, Dong CX, Zhang JJ and Meng XK: TNF- $\alpha$, HGF and TGF- $\beta 1$ are involved in the liver regeneration following partial hepatectomy using portal vein arterializations. J Med Biochem 31: 135-139, 2012.

22. Cuschieri J and Maier RV: Mitogen-activated protein kinase (MAPK). Crit Care Med 33 (12 Suppl): S417-S419, 2005.

(1) (1) This work is licensed under a Creative Commons

Attribution-NonCommercial-NoDerivatives 4.0 International (CC BY-NC-ND 4.0) License. 